\section{ORIGINAL PAPER}

doi: 10.5455/medarh.2016.70.123-126

Med Arch. 2016 Apr; 70(2): 123-126

Received: DEC 15, 2015 | Accepted: JAN 30, 2016

(C) 2016 Cem Cankaya, and Mehmet Tecelloglu

This is an Open Access article distributed under the terms of the Creative Commons Attribution Non-Commercial License

(http://creativecommons.org/licenses/ by-nc/4.0/) which permits unrestricted non-commercial use, distribution, and reproduction in any medium, provided the original work is properly cited.

\title{
Foveal Thickness Alterations in Patients with Migraine
}

\author{
Cem Cankaya ${ }^{\prime}$, and Mehmet Tecellioglu ${ }^{2}$ \\ 'Gozde Kisla Hospital, Eye Clinic, Malatya, Turkey \\ ${ }^{2}$ Department of Neurology, Faculty of Medicine, University of Inonu, Malatya, Turkey
}

Corresponding author: Mehmet Tecellioglu, MD. Department of Neurology, Faculty of medicine, University of Inonu. 44280 Malatya, Turkey. Mob.:+0905337619271. E-mail: mehmettecelli@hotmail.com

\section{ABSTRACT}

Aim: To investigate the alterations in foveal retinal thickness (FT) values in patients with migraine and to reveal the correlations between FT and clinical characteristics of migraine disease. Methods: This study included sixty-eight eyes of 34 migraine patients [twenty-eight eyes of 14 patients with aura( group 1), and forty eyes of 20 patients without aura (group 2)] and forty eyes of 20 healthy volunteer who served as the control group (group $3)$. FT values were measured by optical coherence tomography (OCT) in each group. $\mathbf{R e}-$ sults: Mean age of patients in group 1, 2, and 3 was $34.0 \pm 6.82,35.2 \pm 10.12$, and 35.1 \pm 6.85 years, respectively ( $p=0.84$ ). Mean FT was $211.07 \pm 7.36,220.0 \pm 12.01$, and $221.85 \pm 12.27$ in groups 1, 2, and 3, respectively. There was statistically significance among the group 1-2 and $1-3(p=0.002$ and $p<0.001)$. There was no statistically significance between group 2-3 $(p=0.88)$. Conclusion: This study suggests that in particular migraine with aura may lead to a reduction in FT values. This finding can be explained by the blood flow decrease theory in migraine; however larger studies seem mandatory.

Key words: Foveal thickness; optical coherence tomography; migraine.

\section{INTRODUCTION}

Migraine is a common neurovascular disorder characterized by repeated attacks of severe headache. It can cause intense throbbing or a pulsing sensation in one area of the head and is commonly accompanied by nausea, vomiting, and extreme sensitivity to light and sound. Symptoms typically last 4 to $72 \mathrm{~h}$ and may be severe (1-4).

While some migraine types are preceded or accompanied by transient neurological symptoms (migraine with aura) such as flashes of light, blind spots, or tingling in the arms or legs, others manifest only as a headache (classic migraine). Auras occur in about $25 \%$ of patients, usually just before but sometimes after the headache $(5,6)$.

The pathophysiology of migraine has yet to be known. The disease is often thought to be of neurovascular origin, with research suggesting the pathological mechanism firstly involves the brain which, in turn, affects the blood vessels. Some researchers feel the key mechanism in the pathology is neurological while others believe the vasculature is at the center of the pathology. The serotonin level is also thought to be an important factor (7-9).

Migraines typically occur when blood vessels in the head begin to contract and expand in an abnormal manner. The arteries in the back of the head have been shown to go into spasm, causing a reduced blood flow to the back part of the brain or the occipital lobe. This is thought to trigger aura that are followed by migraine (10).

Optical coherence tomography (OCT) is a noninvasive, noncontact, transpupillary imaging technique that renders in vivo cross sectional view of the retina. OCT utilizes a concept known as inferometry in order to create a cross-sectional map of the retina that is accurate to within at least 10-15 microns. It is increasingly used in the diagnosis and monitoring of retinal diseases including age-related macular degeneration, diabetic retinopathy, and glaucoma. The anatomic layers within the retina can be differentiated and retinal thickness can be measured (11-14).

In previous studies, retinal infarctions caused by retinal artery occlu- 
sions have been reported due to decreased blood flow in migraine patients (15). Retinal nerve fiber layer thickness values can be used for assessment of ganglion cell and retinal nerve fiber damages (15). At this point, in present study we aimed to investigate whether there is an alteration in foveal thickness values due to decreased blood flow and how these alterations affect the visual functions. To investigate the correlations between FT and clinical characteristics of migraine disease also has been aimed.

\section{MATERIALS AND METHODS}

This prospective, observational, cross-sectional study was conducted at the Eye and Neurology clinics at the Gözde Kisla Hospital. All migraine patients were recruited from the Neurology Clinic according to the criteria of the Headache International Society and all healthy volunteers were recruited from the Ophthalmology Clinic.

All the participants were enrolled from a single center. The race of the participants was Caucasian. All participants gave informed consent to the study, and the tenets of the Declaration of Helsinki were followed.

The study included sixty-eight eyes of 34 migraine patients: twenty-eight eyes of 14 patients with aura (group 1 ), and forty eyes of 20 patients without aura (group 2), and forty eyes of 20 healthy volunteer who served as the control group (group 3).

In all cases, the following tests were performed: visual acuity tests, visual field examination using the (30-2) SITA-Fast program (Humphrey-Zeiss), and a complete slit-lamp examination including both anterior and posterior segment.

Eyes with retinal pathology, cataract, any kind of glaucoma, corneal opacity and eyes previously subjected to any kind of ocular surgery or ocular laser treatment were excluded. Patients with systemic disease such as diabetes mellitus, hypertension, cardiovascular and renal diseases and central nervous system disorders were eliminated from the study. Patients with corneal and lens pathologies preventing fundus examination and OCT imaging were also excluded.

All patients and controls who met the inclusion criteria have been examined with the RS-3000 OCT Retina Scan (Nidek Inc., CA, USA), which is a high-speed SD-OCT/confocal ophthalmoscope system. Measurements were repeated until images of $\geq 9 / 10$ quality were obtained. Three measurements were taken from the retina and the average of three measurements was used. Submitted scans were assessed for signal strength index, image centration and color cross section. Signal strength index greater than 50 were included. Scans that were decentered or had poor color cross-sections were excluded.

\section{Statistical assessment}

For the statistical evaluation of the data of the study, SPSS for Windows Version 17.0 (SPSS Inc., Chicago, IL) software was used. Measurable data of the study was presented as mean \pm standard deviation (SD). To assess the correlation of the data with normal distribution, Kolmogorov-Smirnov test was used. Differences among the groups were analyzed by Independent $\mathrm{T}$ test (parametric). One-way ANOVA test was used to compare the groups more than 2. P value less than 0.05 was considered as statistically significant.

\section{RESULTS}

Mean age of patients in group 1, 2, and 3 was $34.0 \pm 6.82$, $35.2 \pm 10.12$, and $35.1 \pm 6.85$ years, respectively. No significant difference was found between the groups regarding age $(\mathrm{p}=0.84)$. Mean FT was 211.07 $\pm 7.36,220.0 \pm 12.01$, and $221.85 \pm 12.27$ in groups 1,2 , and 3, respectively. There was statistically significance among the group 1-2 and $1-3(\mathrm{p}=0.002$ and $\mathrm{p}<0.001)$. There was no statistically significance between group $2-3(\mathrm{p}=0.88)$

\begin{tabular}{lccc}
\hline Features & Group 1 & Group 2 & Group 3 \\
\hline Age, years (mean \pm SD) & $34.0 \pm 6.82$ & $35.2 \pm 10.12$ & $35.1 \pm 6.85$ \\
Gender (F/M) & $8 / 6$ & $12 / 8$ & $10 / 10$ \\
\hline
\end{tabular}

Table 1. Demographic features of the groups

\begin{tabular}{cccc}
\hline & Group 1 & Group 2 & Group 3 \\
& $(n=14)$ & $(n=20)$ & $(n=20)$ \\
\hline FT & $211.07 \pm 7.36^{*}$ & $220.0 \pm 12.01$ & $221.85 \pm 12.27$
\end{tabular}

Table 2. Mean FT values of the groups

\begin{tabular}{ccc}
\hline & Group 1 & Group 2 \\
& $(n=14)$ & $(n=20)$ \\
\hline Diagnosis time (year) & $4.78 \pm 3.1$ & $3.35 \pm 2.46$ \\
Frequency of attacks (month) & $2.28 \pm 1.24$ & $4.4 \pm 1.67$ \\
Length of attack (hour) & $2.85 \pm 1.07$ & $2.55 \pm 1.17$
\end{tabular}

n: number of the patients

Table 3. Clinical characteristics of migraine subgroup

Table 1 shows the demographic features of the groups, Table 2 shows the results of mean FT values in the groups, and Table 3 shows the clinical characteristics of migraine subgroups. FT values altered in migraineurs with aura group when compared with migraineurs without aura and control group $\left(\mathrm{p}_{1=} 0.002\right.$ and $\left.\mathrm{p}_{2}<0.001\right)$. There was no statistical significance between the migraineurs without aura group (group 2) and control group (group 3) for FT values. The Pearson correlation analyses revealed that duration of the disease, length of attack or the frequency of attacks of the patients were not significantly correlated with the reduction in the FRT values $\left(\mathrm{p}_{1=} 0.674, \mathrm{p}_{2=}\right.$ 0.130 , and $\left.\mathrm{p}_{3=} 0.666\right)$.

\section{DISCUSSION}

Many hypotheses about the mechanisms involved in the pathogenesis of migraine disease have been proposed, but the issue is still unclear (16). Neurovascular origin seems one of the most popular and important mechanism involved in the pathogenesis of the disease. According to the neurovascular origin of the disease, some changes in different areas beside the brain such as retinal tissue can be observed or expected due to hypoperfusion. The chronic and recurrent nature of the 
disease may lead to permanent structural abnormalities in the brain and also in the retinal tissue. Alterations due to hypoperfusion in optic nerve head and retina may cause ganglion cell death in those group patients. In view of these findings we aimed to investigate whether there is an alteration in foveal thickness values in patients diagnosed with migraine and if an alteration is present, how this alteration affects the visual functions including visual acuity and visual field examination.

Retinal nerve fiber layer (RNFL) thickness in particular foveal retinal thickness (FT) measurements can be used as a mark in order to assess the ganglion cell damages (17). As a result of ganglion cell damage, a reduction in the thickness of these layers can be expected. At this point, optic coherence tomography (OCT) can be used to assess the RNFL and FT thickness. OCT is a reliable, reproducible and noninvasive imaging technique to assess the retinal diseases.

In our study, beside the FT values the correlations between FT values and clinical characteristics of the disease were also investigated. As a result of present study, a statistically significant reduction in FT values of migraine with aura group was detected. The mean FT values were similar in the migraine without aura and control group. The finding can be explained by the blood flow decrease theory in migraine in particular with aura.

In parallel to the results of our study, previous studies revealed a reduction in thickness in the different quadrants of macula. In a study conducted by Martinez et al., RNFL thickness was significantly lower in temporal quadrant of migraine patients compared to control group (18). In another study conducted by Sorkhabi et al., RNFL thickness was only significantly thinner in nasal quadrant in migraineurs compared to the control group (19). Also Gipponi et al. revealed a reduction in term of RNFL in superior quadrant in migraineurs (20). The mean RNFL thickness for nasal and nasal inferior sectors was significantly thinner in the migraineurs' eyes than in those of the controls in the study conducted by Demircan et al (21). In contrast to the results of above studies, Tan et al. reported that there was no statistically significant difference in measurements between migraine and control group by using scanning laser polarimetry (22). Yulek et al. also revealed no significant differences between the controls and the migraine patients for the retinal thickness in any of the quadrants (23). Unlike other studies, we only measured foveal thickness (central 1 $\mathrm{mm}$ area) in migraine patients with and without aura and we compared the results with healthy controls. Gipponi et al. revealed no difference in the foveal thickness and macular volume measurements in female migraine patients (20). In the study of Demircan et al. the mean macular thickness was not significantly different between the migraineurs and control group (21). Unlike their results, we detected a reduction in the foveal thickness values in migraine with aura group. We also detected that duration of the disease, length of attack or the frequency of attacks of the patients were not significantly correlated with the reduction in the FRT values. In parallel to our results, Sorkhabi et al., Demircan et al. and Gipponi et al. also revealed no correlation between the RNFL thickness and clinical characteristics of migraine disease (19-21).

Studies suggest that there is a stronger vascular risk associated with migraine with aura than with migraine without aura (24). In parallel to the results of these studies, FT values were thinner in migraine with aura group compared to migraine without aura group in our study. The result may be explained by the more decreased blood flow in migraineurs with aura. It means more ganglion cell damage. According to our results, we can say that migraineurs with aura may be at increased risk for possible ischemic events. Unlike our study, Demircan et al. found no differences between RNFL, macula, and choroid thicknesses in migraine with aura and migraine without aura subgroups (21).

Furhermore we examined the visual functions including visual acuity and visual field examination of patients during the study. However, we did not detect any difference in terms of these parameters among the groups. In the study conducted by Demircan et al. there was also no significant difference between the migraineurs and the healthy subjects in term of BCVA (21).

One of the main limitation of our study was number of the participants. Other important main limitation was the medical treatment of patients during examination. 4 patients in group 1 and 6 patients in group 2 were receiving oral medications during our study. Some medications such as triptans known as vasoactive agents may affect the ocular pulsations. Sumatriptan is a selective 5-hydroxytryptamine1d (5-HT1d)-receptor agonist, highly effective in the short-term treatment of migraine headaches (25). 2 patients in group 1 and 2 patients in group 2 were receiving these medications at least once a month. In a study conducted by Schmetterer et al. it was revealed that sumatriptan had no apparent effect in the ophthalmic artery (26). Other medications receiving by our study group were including beta-blockers, ergot alkoloids, and anti-epileptic drugs. We did not detect any study relevant the direct effect of these medications on FT measurements, however these medications may affect the disease activity.

As we know the effects of genetic or genetic-ethnic variations, refractive errors, age, and sex on FT measurements, we excluded the patients who had extraordinary refractive error or axial length. The gender, age, and race of our patients were already similar. Additionally in relation to OCT device, because OCT utilizes light waves, media opacities can interfere with optimal imaging. So the OCT is limited the setting of vitreous hemorrhage, dense cataract or corneal opacity. We already excluded those group of patients. The quality of the images are also dependent on the operator. So three measurements were taken from the retina and the average of three measurements was used. All images were taken by same operator. Also there are possible systematic and random possible sources of error with any imaging technique. We tried to minimize these errors by using the device in optimal conditions. 


\section{CONCLUSION}

Our findings suggest that FT values may alter in migraineurs with aura. However, the parameter did not alter in migraineurs without aura and controls. Therefore we can say that according to neurovascular theory in the etiology of migraine disease, decreased blood flow may lead to some structural abnormalities in retinal tissue beside the brain in particular migraineurs with aura. These alterations can be detected by OCT. However the visual functions were not affected from the alterations in retinal tissue. Further studies are needed to confirm the relevance and usefulness of our results.

- Author's contribution: Both authors were included in all phases of preparing this article, including final proof reading.

- Acknowledgments: The authors have no proprietary or commercial interest in any materials discussed in this article.

\section{REFERENCES}

1. Friberg L, Olesen J, Lassen NA, Olsen TS, Karle A. Cerebral oxygen extraction, oxygen consumption, and regional cerebral blood flow during the aura phase of migraine. Stroke. 1994; 25(5): 974-9.

2. Steiner TJ, Stovner LJ, Birbeck GL. Migraine: the seventh disabler. Headache. 2013; 53(2): 227-9.

3. Ripa P, Ornello R, Degan D, Tiseo C, Stewart J, Pistoia F, Carolei A, Sacco S. Migraine in menopausal women: a systematic review. Int J Womens Health. 2015; 20(7): 773-82.

4. Malone CD, Bhowmick A, Wachholtz AB. Migraine: treatments, comorbidities, and quality of life, in the USA. J Pain Res. 2015; 12(8): 537-47.

5. Sharma P, Sridhar J, Mehta S. Flashes and Floaters. Prim Care. 2015; 42(3): 425-35.

6. Charles A, Hansen JM. Migraine-aura: new ideas about cause, classification and clinical significance. Curr Opin Neurol 2015; 28(3): 255-60.

7. Burstein R, Noseda R, Borsook D. Migraine: multiple processes, complex pathophysiology. J Neurosci. 2015; 35(17): 6619-29.

8. Goadsby PJ, Charbit AR, Andreou AP, Akerman S, Holland PR. Neurobiology of migraine. Neuroscience. 2009; 161(2): 327-41.

9. Cutrer FM. Pathophysiology of migraine. Semin Neurol. 2010; 30(2): 120-30.

10. Tietjen GE. Migraine as a systemic vasculopathy. Cephalalgia. 2009; 29(9): 989-96.

11. Konno S, Akiba J, Yoshida A. Retinal thickness measurements with optical coherence tomography and the scanning retinal thickness analyzer. Retina. 2001; 21: 57-61.
12. Wexler A, Sand T, Elsas TB. Macular thickness measurements in heathy Norwegian volunteers: an optical coherence tomography study. BMC Ophthalmol. 2010; 13: 10-13.

13. Figurska M, Robaszkiewicz J, Wierzbowska J. Optical coherence tomography in imaging of macular diseases. Klin Oczna. 2010; 112(4-6): 138-46.

14. Trick GL, Calotti FY, Skarf B. Advances in imaging of the optic dics and retinal nerve fiber layer. J Neuroophthalmol. 2006; 26(4): 284-95.

15. Beversdorf D, Stommel E, Allen.C, Stevens R, Lessell S. Recurrent branch retinal infarcts in association with migraine. Headache. 1997; 37(6): 396-9.

16. Burgos-Vega C, Moy J, Dussor G. Meningeal afferent signaling and the pathophysiology of migraine. Prog Mol Biol Transl Sci. 2015; 131: 537-64.

17. Pagliara MM, Lepore D, Balestrazzi E. The role of OCT in glaucoma management. Prog Brain Res. 2008; 173: 139-48.

18. Martinez A, Proupim N, Sanchez M. Retinal nerve fiber layer thickness measurements using optical coherence tomography in migraine patients. Br J Ophthalmol. 2008; 92(8): 1069-75.

19. Sorkhabi R, Mostafaei S, Ahoor M, Talebi M. Evaluation of retinal nerve fiber layer thickness in migraine. Iran J Neurol. 2013; 12(2): 51-5.

20. Gipponi S, Scaroni N, Venturelli E, Forbice E, Rao R, Liberini P, Padovani A, Semeraro F. Reduction in retinal nerve fiber layer thickness in migraine patients. Neurological Sciences. 2013; 34(6): 841-5.

21. Demircan S, Ataş M, Yuksel SA, Ulusoy MD, Yuvacı I, Arifoglu HB, Başkan B, Zararsız G. The impact of migraine on posterior ocular structures. J Ophthalmol. 2015; 2015: 868967.

22. Tan FU, Akarsu C, Gullu R. Retinal nerve fiber layer thickness is unaffected in migraine patients. Acta Neurol Scand. 2005; 112(1): 19-23.

23. Yulek F, Dirik EB, Eren Y, Simavlı H, Ugurlu N, Cagıl N, Simsek S. Macula and retinal nerve fiber layer in migraine patients: analysis by spectral domain optic coherence tomography. Semin Ophthalmol. 2015; 30(2): 124-8.

24. Sacco S, Ricci S, Carolei A. Migraine and vascular diseases: a review of the evidence and potential implications for management. Cephalalgia. 2012; 32(10): 785-95.

25. Tokuoka K, Takayanagi R, Toyabe M, Watanabe M, Kitagawa Y, Yamada Y. Theoretical analysis of headache recurrence in patients administered triptans for migraine-based on receptor occupancy. J Headache Pain. 2015; 16: 71.

26. Schmetterer L, Wolzt M, Krejcy K, Graselli U, Findl O, Eichler HG, Singer EA. Cerebral and ocular hemodynamic effects of sumatriptan in the nitroglycerin headache model. Clin Pharmacol and Ther. 1996; 60(2): 199-205. 